\title{
The Local Immunity of the Oral Cavity in Women with Recurrent Aphthous Stomatitis Associated with Urogenital Infection
}

\author{
Olga A. Uspenskaya1, Larisa N. Kazarina², Elena A. Shevchenko3, Josef Herbert ${ }^{4}$ \\ ${ }^{1}$ Department of Therapeutic Stomatology, Federal State Budgetary Educational Institution of Higher Education «Privolzhsky \\ Research Medical University» of the Ministry of Health of the Russian Federation, Nizhny Novgorod, Russia \\ ${ }^{2}$ Department of Propaedeutic Dentistry, Federal State Budgetary Educational Institution of Higher Education «Privolzhsky \\ Research Medical University» of the Ministry of Health of the Russian Federation, Nizhny Novgorod, Russia \\ ${ }^{3}$ Department of Pathological Physiology, Federal State Budgetary Educational Institution of Higher Education «Privolzhsky \\ Research Medical University» of the Ministry of Health of the Russian Federation, Nizhny Novgorod, Russia \\ ${ }^{4}$ Dentist Running in Own Practice, Schönebeck, Germany \\ Email: uspen ska ya.olga2011@yandex.ru
}

How to cite this paper: Uspenskaya, O.A., Kazarina, L.N., Shevchenko, E.A. and Herbert, J. (2019) The Local Immunity of the Oral Cavity in Women with Recurrent Aphthous Stomatitis Associated with Urogenital Infection. Open Journal of Stomatology, 9, 1-8.

https://doi.org/10.4236/ojst.2019.91001

Received: July 12, 2018

Accepted: December 22, 2018

Published: December 25, 2018

Copyright $\odot 2019$ by authors and Scientific Research Publishing Inc. This work is licensed under the Creative Commons Attribution International License (CC BY 4.0).

http://creativecommons.org/licenses/by/4.0/

\begin{abstract}
This paper studied the dynamics of local immunity factors of the oral cavity in women with recurrent aphthous stomatitis against the background of urogenital infection. The research proves the maximum efficacy of hydroxyzine hydrochloride, aminodihydrophthalasindione sodium and Eplan used in multiple treatments of recurrent aphthous stomatitis in patients with urogenital infection as evidenced by a marked reduction in the coefficient of local immunity factors balance, which is observed as early as on day 7 of the therapy provided. Moreover, the findings show the growth of immunological parameters of oral fluid (lysozyme and SIgA), the normalization of the coefficient of local immunity factors balance in the treatment of recurrent aphthous stomatitis in women with urogenital infection, the improvement being observed 3 months after the treatment suggesting a favorable local immunity of the oral cavity.
\end{abstract}

\section{Keywords}

Hydroxyzine Hydrochloride, Aminodihydrophthalasindione Sodium, Eplan, Chronic Recurrent Aphthous Stomatitis, Coefficient of Local Immunity Factors Balance, Secretory IgA, Lysozyme, Immunoglobulin A, G

\section{Introduction}

Recurrent aphthous stomatitis (RAS) is frequent in dental practice [1] [2] [3] [4] 
[5]. The disease is long lasting and characterized by frequent recurrences, resistance to the treatment provided [1] [4] [6]. Some scientists distinguish a variety of etiological factors, among them there are immune, neurogenic, infection-allergic and other theories of RAS origin [1] [2] [5]. Reports in literature give a variety of different techniques and medications for RAS treatment. However, RAS cure is still an urgent problem [1] [2] [3] [4] [5] [7].

In this regard, our study aimed at optimizing the methods used to treat chronic recurrent aphthous stomatitis (CRAS). To accomplish the purpose, we set a problem of proving the use of some medications in RAS therapy based on the study of the changes; a number of immunological parameters of oral fluid undergo during the treatment.

\section{Materials and Methods}

The present study was carried out at the Department of Therapeutic Dentistry, Nizhny Novgorod State Medical Academy for 12 years. The study included data from a survey of 1000 women aged 25 - 35 years with chronic recurrent aphthous stomatitis, 500 practically healthy women were included in the control group.

Among them, groups of patients with chronic recurrent aphthous stomatitis (CRAS) were identified who applied to the dental clinic NizhGMA, MLPU "Women's Consultation No. 5" in Nizhny Novgorod, the NOCC, the Research Institute of Preventive Medicine NizhGMA, OC AIDS with complaints of pain in the SDR and from the urogenital tract reproductive problems, as well as for preventive examination. We performed a clinical examination of 300 women with RAS associated with urogenital infection, aged from 25 to 35 years (mean age $28.2 \pm 1.4)$ followed by a treatment. Exclusion criteria were severe somatic diseases (diabetes, HIV, cancer, blood diseases, etc.), refusal of examination and treatment, age to 25 and after 35 years. Eligibility criteria are women from 25 to 35 years old with gynecological diseases and CRAS.

CRAS patients were randomized in tree groups, 100 women in each group, depending on the treatment techniques used: group I (main group) with drug therapy including general treatment combined with therapeutic agents: hydroxyzine hydrochloride, aminodihydrophthalasindione sodium, and local treatment: Eplan applied on problem oral mucosa areas; group II with drug therapy consisting in general treatment combined with therapeutic agents: hydroxyzine hydrochloride, aminodihydrophthalasindione sodium, and local treatment-problem areas of oral mucosa were covered by solcoseryl (dental adhesive paste); and group III with drug therapy including general treatment with antihistamines and multivitamin preparations administered, and local treatment: solcoseryl (dental adhesive paste) applied on problem oral mucosa areas.

Patients of all 3 groups (100 in each) with CRAS for anamnestic data, complaints for primary treatment, age, baseline values of dental and gynecological status were comparable. Patients of the examined groups were examined hor- 
monal and immune status, biochemical and general blood test, determination of blood groups and $\mathrm{Rh}$ factor. A comparative evaluation of various diagnostic techniques was carried out, in particular, the reaction of direct immunofluorescence and polymerase chain reaction in real time, enzyme immunoassay, bacteriological culture. All patients received informed consent to participate in the study and receive Galavit, Atarax and Eplan in the treatment of CRAS, as well as the permission of the Ethics Committee.

The study included several items:

1) Dental examination of women, diagnosis of CRAS, the study of the occurrence of dental diseases in women with gynecological pathology evaluation of dental status in women with gynecological pathology.

2) General clinical methods of examination.

3) Study of the features of the etiology of urogenital infection in prolific and infertile women with CRAS, combination of dental pathology with infections of the urogenital tract.

4) Conducting a comparative evaluation of the immune status in prolific and infertile patients of CRAS.

5) Study of hormonal status in patients with CRAS in combination with urogenital infection and infertility.

6) Detection of the nuances of fluctuations in changes in the oral fluid and blood during dental pathology against the background of gynecological diseases.

Aminodihydrophthalasindione sodium was administered in the form of sublingual tablets according to the scheme: daily for 10 days, 4 tablets per $24 \mathrm{~h}$, and during the following 10 days every other day at the same dose. Thus, the therapy regimen was 30 days. Hydroxyzine hydrochloride was given t.i.d.: by $12.5 \mathrm{mg}$ in the morning and afternoon, and $25 \mathrm{mg}$ in the evening within 4 weeks. The oral cavity was treated by antiseptics followed by Eplan applications for $20-30 \mathrm{mi}-$ nutes 3 - 4 times a day until the epithelialization of affected areas. The patients were followed up for 12 months after the therapy provided. This scheme is generally accepted and its effectiveness is proved in use, as is shown in the annotation to the drug and publications.

During the laboratory stage we determined the level of secretory immunoglobulin A (SIgA) by radial immunodiffusion (RID) in gel-G. Mancini, A. Carbonara (1965) using guidelines by E.V. Tchernokhvostova, S.I. Golderman (1975); serum immunoglobulins (G. A) in oral cavity using radial immunodiffusion (RID) in gel (G. Mancini, A. Carbonara, 1965); lysozyme in oral cavity by photonephelometry (V. G. Dorofeichuk, 1968), as well as a coefficient of local immunity factors balance $\left(\mathrm{C}_{\mathrm{b}}\right)$ developed by V.G. Dorofeichuk and Tolkacheva et al. (1987) [8].

$\mathrm{C}_{\mathrm{b}}$ was used for an integral assessment of local oral immunity. $\mathrm{C}_{\mathrm{b}}$ formula was devised considering the functional relationships of lysozyme with IgG and IgA.

$$
C_{B}=\frac{\operatorname{IgG} \cdot 40 \%}{\operatorname{Ig} A \cdot 0.6 \cdot l a}
$$


where:

$\operatorname{Ig} G, \operatorname{Ig} A$ is concentration of immunoglobulins;

la is lysozyme activity in secretion;

$40 \%$ is conditional norm of lysozyme activity;

0.6 is $\operatorname{Ig} G / \operatorname{Ig} A$ relationship, which was true in most healthy people.

For the present study we used oral fluid samples, 3 - $5 \mathrm{ml}$, taken in the morning, fasting, with no stimulation, and kept in a fridge at $-20^{\circ} \mathrm{C}$ till the laboratory stage.

The findings were statistically processed using the methods for findings reliability assessment, analysis of variance, and autocorrelation according to standard techniques. The obtained data were processed and analyzed using Microsoft Office (Excel) application programs, and a software statistical package "Stadia" and "Statistica 7.0".

\section{Results}

Table 1 shows the findings of immunological parameters of oral fluid in the treatment of 300 patients with RAS and urogenital infection, and 50 apparently healthy subjects (control).

The represented data show a slight IgA decrease in the examined women compared to controls, on day 7 after the treatment there was a significant decrease of IgA level ( $\mathrm{p} \leq 0.05)$, with the following increase by month 3 .

IgG amount was lower in the examined women compared to those of the control group, and decreased sharply by month 3 ( $\mathrm{p} \leq 0.05$ ).

Lysozyme level was significantly reduced in RAS patients compared to the controls $(\mathrm{p} \leq 0.05)$. Significant lysozyme decrease was found by day 7 after therapy ( $\mathrm{p} \leq 0.05)$, while on day 90 its level was significantly higher than the initial one.

In patients with RAS and urogenital infection $C_{b}$ was significantly higher compared to the controls $(\mathrm{p} \leq 0.05)$ that argued for moderate local immunity of the oral cavity. By day 7 after therapy $C_{b}$ slightly decreased, still remaining significantly higher compared to that in controls $(\mathrm{p} \leq 0.05)$. $\mathrm{C}_{\mathrm{b}}$ sharply decreased by month 3 after therapy $(\mathrm{p} \leq 0.05)$ reaching the values similar to those in the control group ( $\mathrm{p} \leq 0.05)$, i.e., there was favorable local immunity of the oral cavity.

SIgA level in RAS patients was significantly higher compared to controls, on day 7 and day 90 after therapy SIgA was found to have a significant increase ( $\mathrm{p} \leq$ 0.05), it approaching the values in the control group.

Thus, a significant increase of immunological parameters of the oral fluid (IgG, SIgA, $\mathrm{C}_{\mathrm{b}}$ ) was found in the treatment of recurrent aphthous stomatitis in women with urogenital infection, the increase being the most significant on month 3 after therapy.

The analysis of $C_{\mathrm{b}}$ changes in the oral fluid depending on a treatment method showed the following. 
Table 1. Immunological parameters of oral fluid in recurrent aphthous stomatitis and urogenital infection $(\mathrm{N}=350)$.

\begin{tabular}{ccccc}
\hline & \multicolumn{4}{c}{ Time periods before and after therapy (days) } \\
\cline { 2 - 5 } Oral fluid parameters & Control group & Before therapy & \multicolumn{3}{c}{ } & 90 \\
\cline { 2 - 5 } & \multicolumn{4}{c}{$\mathrm{M} \pm \mathrm{m}$} \\
\hline IgA, g/l & $0.06 \pm 0.002$ & $0.058 \pm 0.003$ & $0.041 \pm 0.002^{*}$ & $0.053 \pm 0.002$ \\
IgG, g/l & $0.08 \pm 0003$ & $0.065 \pm 0.008$ & $0.06 \pm 0.01$ & $0.033 \pm 0.004 *$ \\
Lysozyme, \% & $45.2 \pm 2.3$ & $32.6 \pm 1.4^{*}$ & $25.3 \pm 1.2^{*}$ & $37.0 \pm 2.2^{*}$ \\
$\mathrm{C}_{\mathrm{b}}$ & $1.54 \pm 0.17$ & $4.595 \pm 0.543^{*}$ & $3.258 \pm 0.423^{*}$ & $1.534 \pm 0.184$ \\
SIgA, g/l & $0.33 \pm 0.09$ & $0.089 \pm 0.012^{*}$ & $0.125 \pm 0.002$ & $0.242 \pm 0.013$ \\
\hline
\end{tabular}

*_-significant differences in relation to controls $(\mathrm{p}<0.05)$.

Group II demonstrated $C_{b}$ downtrend on day 7. By month 3 and month $6 \mathrm{C}_{\mathrm{b}}$ in this group was significantly lower than the initial value $(\mathrm{p} \leq 0.05)$, on month 12 it remained stable, its value being significantly lower than that before the treatment $(\mathrm{p} \leq 0.05)$. Group I was found to have $\mathrm{C}_{\mathrm{b}}$ decrease on day 7 . On month 3 the parameter's value went down significantly compared to its level before the therapy ( $\mathrm{p} \leq 0.05$ ); by month 6 there was a slight growth of $\mathrm{C}_{\mathrm{b}}$ level, while by month 12 it decreased again remaining lower than that before the treatment $(\mathrm{p} \leq 0.05)$.

In group III $C_{b}$ level slightly decreased by day 7 and by day 90 . On day 180 we observed its growth $(\mathrm{p} \leq 0.05)$, however, 12 months after the treatment $\mathrm{C}_{\mathrm{b}}$ level significantly decreased $(\mathrm{p} \leq 0.05)$.

During all the periods of observation $\mathrm{C}_{\mathrm{b}}$ values were nearly the same in groups I and II, $\mathrm{C}_{\mathrm{b}}$ decrease being accentuated in group I.

Thus, RAS therapy resulted in $\mathrm{C}_{\mathrm{b}}$ decrease in all 3 groups, the maximum effect was found in group I as early as on day 7 after the treatment initiation.

\section{Discussion}

There are a lot of reviews on RAS [4] [6] [9] [10] [11] [12]. A number of various treatment strategies has been evaluated using drug therapy, topical agents and mouth washes [13] [14] [15] [16] [17]. In studies laser therapy is one of the suggested treatments to reduce patients' discomfort [10] [15] [18]. In another study the application of ozone on RAS lesions was used for treatment [19].

In the literature available to us and to the best of our knowledge, this study is the first of such type, which demonstrates the local immunity of the oral cavity in women with RAS associated with urogenital infection.

In the present examination a significant increase in the immunological indices of the oral fluid (IgG, SIgA, Xb.) was observed in the treatment of patients with aphthous stomatitis and urogenital infection with the scheme proposed by us, using atarax, eplan and galavit, most pronounced at 3 months of treatment. These changes may be associated with the effect of the drug's use. 
So, the mechanism of Galavit action is associated with its ability to regulate the functional and metabolic activity of innate and adaptive immunity (monocytes, macrophages, neutrophils, natural killers, and others). Galavit normalizes the phagocytic activity of monocytes/macrophages, the neutrophils bactericidal activity and the cytotoxic activity of NK cells. At the same time, restoring the reduced activity of immune cells, the drug increases the body's resistance to infectious diseases of bacterial, viral and fungal etiology, contributes to a more rapid elimination of the pathogen from the body, and reduces the frequency, severity and duration of infections. In addition, Galavit ${ }^{\oplus}$ normalizes antibody production, increases the functional antibodies activity (affinity), indirectly regulates the production of endogenous interferons (IFN- $\alpha$, IFN- $\gamma$ ) by producing cells. In inflammatory diseases, the drug reversibly (for $6-8$ hours) inhibits the excessive synthesis of hyperactive macrophages of tumor necrosis factor- $\alpha$, interleukin-1, interleukin- 6 and other proinflammatory cytokines, the level of which determines the degree of inflammatory reactions, their cyclicity, as well as the severity of intoxication of the organism. Galavit ${ }^{\circledR}$ reduces the production of reactive oxygen species by hyperactive macrophages, thereby reducing the level of oxidative stress and protecting tissues and organs from the damaging effects of radicals. Normalization of excessively increased functional activity of phagocytic cells leads to the restoration of their antigen presenting and regulating function, reducing the level of auto-aggression.

Atarax (a derivative of diphenylmethane), has a moderate anxiolytic activity; also has a sedative, antiemetic, antihistamine and $\mathrm{m}$-anticholinergic action. It blocks central $\mathrm{m}$-cholinergic and histamine $\mathrm{H} 1$ receptors and inhibits the certain subcortical zones activity.

Eplan has a bactericidal, anti-inflammatory, wound-healing, regenerating and analgesic effect, which is provided by the active ingredients of the drug.

Thus, the combined use of these drugs has an impact on different pathogenesis of recurrent aphthous stomatitis (RAS), leading, inter alia, to an increase in the immunological resistance.

\section{Conclusion}

The study carried out proves maximum efficiency of hydroxyzine hydrochloride, aminodihydrophthalasindione sodium, and Eplan (group I) used in the combination therapy of RAS in women with urogenital infection as evidenced by significant $\mathrm{C}_{\mathrm{b}}$ decrease in group I as early as on day 7 of therapy. Moreover, the findings suggest the increase of immunological parameters of the oral fluid (lysozyme and SIgA), the normalization of $\mathrm{C}_{\mathrm{b}}$ values during the treatment of recurrent aphthous stomatitis in women with urogenital infection, the normalization being the most significant on month 3 after therapy, indicating a favourable local immunity of the oral cavity.

\section{Conflicts of Interest}

The authors declare no conflicts of interest regarding the publication of this paper. 


\section{References}

[1] Shevchenko, E.A. (2011) Alteration Peculiarities of Some Biochemical Blood Parameters in Viral Urogenital Infections. Virology Issues, 56, 39-41.

[2] Shevchenko, E.A. and Uspenskaya, O.A. (2012) The Study of Interactions of the Factors Contributing to Persistence Development in Urogenital Infections. Pathologic Physiology and Experimental Therapy, 56, 57-59.

[3] Akintoye, S.O. and Greenberg, M.S. (2014) Recurrent Aphthous Stomatitis. Dental Clinics of North America, 58, 281-297. https://doi.org/10.1016/j.cden.2013.12.002

[4] Belenguer-Guallar, I., Jimenez-Soriano, Y. and Claramunt-Lozano, A. (2014) Treatment of Recurrent Aphthous Stomatitis. A Literature Review. Journal of Clinical and Experimental Dentistry, 6, e168-e174. https://doi.org/10.4317/jced.51401

[5] Shevchenko, E.A., Uspenskaya, O.A., Kondyurov, I.M., Kurylev, V.V. and Rossokhin, V.F. (2012) Assessment of Viral Component for the Purpose of Diagnosing and Treating Inflammatory Oral Diseases. Modern Technologies in Medicine, 4, 96-99.

[6] Slebioda, Z., Szponar, E. and Kowalska, A. (2014) Etiopathogenesis of Recurrent Aphthous Stomatitis and the Role of Immunologic Aspects: Literature Review. Archivum Immunologiae et Therapiae Experimentalis, 62, 205-215. https://doi.org/10.1007/s00005-013-0261-y

[7] Kazarina, L.N. and Pursanova, A.E. (2014) Clinicoimmunological Assessment of Efficient Usage Polyoxidonium and Tantum Verde in Catarrhal Gingivitis Treatment in Children with Chronic Gastroduodenitis. Dentistry, 1, 43-45.

[8] Karpistchenko, A.L. (1999) Medical Laboratory Technologies and Diagnostics: A Reference Book. Intermedika, S. Petersburg.

[9] Tarakji, B., Gazal, G., Al-Maweri, S.A., Azzeghaiby, S.N. and Alaizari, N. (2015) Guideline for the Diagnosis and Treatment of Recurrent Aphthous Stomatitis for Dental Practitioners. Journal of International Oral Health, 7, 74-80.

[10] Han, M., Fang, H., Li, Q.L., Cao, Y., Xia, R. and Zhang, Z.H. (2016) Effectiveness of Laser Therapy in the Management of Recurrent Aphthous Stomatitis: A Systematic Review. Scientifica, 2016, Article No. 9062430. https://doi.org/10.1155/2016/9062430

[11] Ranganath, S.P. and Pai, A. (2016) Is Optimal Management of Recurrent Aphthous Stomatitis Possible? A Reality Check. Journal of Clinical and Diagnostic Research, 10, Ze08-Ze13. https://doi.org/10.7860/JCDR/2016/19519.8643

[12] Edgar, N.R., Saleh, D. and Miller, R.A. (2017) Recurrent Aphthous Stomatitis: A Review. Journal of Clinical and Aesthetic Dermatology, 10, 26-36.

[13] Abbasi, F., Raoof, M., Khatami, R., Shadman, N., Borjian-Boroojeni, F. and Nazari, F. (2016) Effectiveness of Amlexanox and Adcortyl for the Treatment of Recurrent Aphthous Ulcers. Journal of Clinical and Experimental Dentistry, 8, e368-e372. https://doi.org/10.4317/jced.52540

[14] Mehdipour, M., Taghavi Zenooz, A., Sohrabi, A., Gholizadeh, N., Bahramian, A. and Jamali, Z. (2016) A Comparison of the Effect of Triamcinolone Ointment and Mouthwash with or without Zinc on the Healing Process of Aphthous Stomatitis Lesions. Journal of Dental Research Dental Clinics Dental Prospects, 2, 87-91. https://doi.org/10.15171/joddd.2016.014

[15] Nasry, S.A., El Shenawy, H.M., Mostafa, D. and Ammar, N.M. (2016) Different Modalities for Treatment of Recurrent Aphthous Stomatitis. A Randomized Clinical Trial. Journal of Clinical and Experimental Dentistry, 8, e517-e522. https://doi.org/10.4317/jced.52877 
[16] Ofluoglu, D., Ergun, S., Warnakulasuriya, S., Namdar-Pekiner, F. and Tanyeri, H. (2017) An Evaluation of the Efficacy of a Topical Gel with Triester Glycerol Oxide (TGO) in the Treatment of Minor Recurrent Aphthous Stomatitis in a Turkish Cohort: A Randomized, Double-Blind, Placebo-Controlled Clinical Trial. Medicina oral, patologia oral y cirugia bucal, 2, e159-e166. https://doi.org/10.4317/medoral.21469

[17] Rahmani, F., Moghadamnia, A.A., Kazemi, S., Shirzad, A. and Motallebnejad, M. (2018) Effect of $0.5 \%$ Chitosan Mouthwash on Recurrent Aphthous Stomatitis: A Randomized Double-Blind Crossover Clinical Trial. Electron Physician, 6, 6912-6919. https://doi.org/10.19082/6912

[18] Zeini Jahromi, N., Ghapanchi, J., Pourshahidi, S., Zahed, M. and Ebrahimi, H. (2017) Clinical Evaluation of High and Low-Level Laser Treatment (CO2vsInGaAlP Diode Laser) for Recurrent Aphthous Stomatitis. J Dent (Shiraz), 18, 17-23. https://www.ncbi.nlm.nih.gov/pubmed/

[19] Al-Omiri, M.K., Alhijawi, M., AlZarea, B.K., Abul Hassan, R.S. and Lynch, E. (2016) Ozone Treatment of Recurrent Aphthous Stomatitis: A Double Blinded Study. Scientific Reports, 6, Article Number: 27772. https://doi.org/10.1038/srep27772 